\title{
Linear Free Energy Relationship Correlation of the Distribution of Solutes between Water and Sodium Dodecyl Sulfate (SDS) Micelles and between Gas and SDS Micelles
}

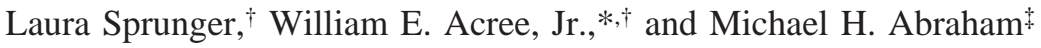 \\ Department of Chemistry, P.O. Box 305070, University of North Texas, Denton, Texas 76203-5070, and \\ Department of Chemistry, University College London, 20 Gordon Street, London WC1H 0AJ, U.K.
}

Received May 22, 2007

\begin{abstract}
Data have been assembled from the published literature on the water-to-micellar sodium dodecyl sulfate (SDS) partition coefficient data for more than 200 compounds and on the gas-to-micellar SDS partition coefficient data for more than 140 compounds. It is shown that an Abraham solvation equation with only five descriptors can be used to correlate the observed partition coefficient data to within a standard deviation of $0.22 \mathrm{log}$ units. Micellar electrokinetic chromatographic (MEKC) retention factor data measured on a micellar SDS pseudostationary phase was also gathered from the literature. The water-to-micellar SDS partition coefficient and MEKC retention factor data were combined into a single database and correlated with the Abraham model. The derived correlation described the 486 experimental values to within a standard deviation of $0.15 \log$ units. The micellar SDS system has been compared to various liquid phases in terms of solubility of gases and vapors and has been shown to be a very selective system-more so than room-temperature ionic liquids.
\end{abstract}

\section{INTRODUCTION}

The contamination of soils, sediments, and groundwater by hazardous organic materials is a widespread environmental concern. Hydrophobic organic contaminants (HOCs) are of particular interest because such compounds are strongly sorbed to soils/sediments, and remediation through desorption is often a very expensive, time-consuming process due to the compounds' low aqueous solubilities. Surfactant enhanced remediation has been suggested as a viable means for removal of adsorbed HOCs from soils and sediments. Published studies have shown that enhanced aqueous solubilization of HOC is achieved in the presence of dissolved surfactants, particularly at surfactant concentrations above their critical micelle concentration ( $\mathrm{cmc}$ ). The enhanced solubilization phenomena observed in aqueous micellar solutions not only is important in desorption of organic pollutants from soils/sediments but also is used in numerous industrial processes, such as protein purification, micellar catalysis, and drug product formulation.

The enhanced solubility is due to the partitioning of the organic solute into the micellar phase. Solute transfer from the bulk aqueous phase to the micellar region is governed by a partition coefficient, which can be mathematically described in either mole fraction concentration units

$$
P_{\mathrm{x}}=\frac{\text { mole fraction of solute in micellar phase }}{\text { mole fraction of solute in aquous phase }}
$$

or molar concentrations

$$
P_{\mathrm{c}}=\frac{\text { molarity of solute in micellar phase }}{\text { molarity of solute in aquous phase }}
$$

\footnotetext{
* Corresponding author e-mail: acree@unt.edu.

$\dagger$ University of North Texas.

$\doteqdot$ University College London.
}

where the solute concentrations in the corresponding phases are measured near infinite dilution. Sepulveda et al. ${ }^{1}$ reviewed several experimental solubility and spectroscopic methods that are used to measure micelle-water partition coefficients. The authors compiled available partition coefficient data for solutes in aqueous micellar sodium dodecyl sulfate (SDS), hexadecyltrimethylammonium bromide (CTAB), dodecyltrimethylammonium bromide (DTAB), and hexadecyltrimethylammonium chloride solutions. Additional data compilations are available for sodium dodecyl sulfate ${ }^{2}$ and hexadecylpyridinium chloride ${ }^{3}$ micellar solution. Each published correlation contains experimental data for fewer than 200 solutes per surfactant.

To address the scarcity of measured aqueous-micellar partition coefficient data, researchers have turned to predictive methods as a means to generate desired values. Valsaraj and Thibodeaux ${ }^{4}$ observed that there was a good correlation between the logarithm of the molar SDS-water partition coefficient $\log P_{\mathrm{c}, \mathrm{SDS} / \text { water, }}$ and the logarithm of the 1-octanol/ water partition coefficient, $\log P_{\text {ОтОН/water }}$

$$
\begin{aligned}
\log P_{\mathrm{c}, \mathrm{SDS} / \text { water }} & =0.32+0.827 \log P_{\mathrm{OTOH} / \mathrm{water}} \\
(N & =57, R=0.9924)
\end{aligned}
$$

whenever aliphatic amides and lactams were excluded. Abraham et al. later reanalyzed all of the partition coefficient data and found

$$
\begin{gathered}
\log P_{\mathrm{c}, \mathrm{SDS} / \text { water }}=0.740+0.693 \log P_{\mathrm{OTOH} / \text { water }} \\
(N=63, R=0.9224, \mathrm{SD}=0.38, F=348)
\end{gathered}
$$

where $N$ denotes the number of data points, $R$ is the correlation coefficient, SD refers to the standard deviation, and $F$ is the Fisher F-statistic. Valsaraj and Thibodeaux also developed a bond contribution method as well as a group 
contribution method for estimating the water-SDS partition coefficients. The authors did not provide any indication of the deviations between the observed partition coefficients and calculated values based on the latter two methods.

Abraham et al. ${ }^{2}$ reported a linear free energy relationship (LFER)

$$
\begin{aligned}
& \log P_{\mathrm{x}, \mathrm{SDS} / \text { water }}=1.280(0.060)+0.484(0.063) \mathbf{E}- \\
& 0.431(0.079) \mathbf{S}-0.183(0.067) \mathbf{A}-1.721(0.088) \mathbf{B}^{\circ}+
\end{aligned}
$$$$
2.878(0.079) \mathbf{V}(5)
$$

$$
(N=138, R=0.9808, \mathrm{SD}=0.192, F=668)
$$

based on the mole fraction partition coefficient data of 138 assorted compounds at $298 \mathrm{~K}$. The independent variables in eq 5 are solute descriptors as follows: $\mathbf{E}$ is an excess molar refraction, $\mathbf{S}$ is the dipolarity/polarizability, $\mathbf{A}$ and $\mathbf{B}^{\circ}$ are the hydrogen-bond acidity and basicity, respectively, and $\mathbf{V}$ is the McGowan molecular volume. The alternative basicity solute descriptor, $\mathbf{B}^{\circ}$, was used in eq 5 because the micellar phase contains an appreciable amount water. For most solutes the two Abraham basicity solute descriptors, $\mathbf{B}$ and $\mathbf{B}^{\circ}$, are numerically equivalent. The values differ for only a few solutes, most notably for sulfoxides, anilines, and alkylpyridines.

Bel'skii ${ }^{5}$ later applied the Abraham model to describe the distribution of nonionic compounds between the gas phase and sodium dodecyl sulfate micelles, $K_{\mathrm{SDS} / \mathrm{gas}}$

$$
\begin{gathered}
\log K_{\mathrm{SDS} / \mathrm{gas}}=-0.947(0.112)+0.392(0.107) \mathbf{E}+ \\
2.027(0.140) \mathbf{S}+3.834(0.144) \mathbf{A}+2.242(0.182) \mathbf{B}+ \\
2.869(0.123) \mathbf{V}(6) \\
(N=104, R=0.990, \mathrm{SD}=0.310)
\end{gathered}
$$

While the statistics of eq 6 are quite good, the authors used the wrong form of the Abraham model to describe solute transfer from the gas phase. The Abraham model correlation for solute transfer from the gas phase contains the $\mathbf{L}$ descriptor ( $\mathbf{L}$ is the logarithm of the solute gas-phase dimensionless Ostwald partition coefficient into hexadecane at $298 \mathrm{~K}$ ), rather than the McGowan molecular volume descriptor.

In the present study we have re-examined the Abraham model correlation for aqueous-micellar SDS partition coefficients using a much larger database. Through a search of the chemical literature we found experimental $P_{\mathrm{x}, \mathrm{SDS} / \text { water }}$ values for 214 different compounds, which is considerably more than the 138 compounds that Abraham et al. ${ }^{2}$ used in deriving eq 5 . As part of the present study we have developed a correlation equation for the gas-to-aqueous SDS micellar phase partition coefficient based on the Abraham model LFER expression for gas to condensed phase transfer. The correlation reported by Bel'skii, eq 6, used the form of the Abraham model for describing solute transfer between two condensed phases. While the statistics of the Bel'skii correlation are quite good, it is desirable to have the $\log$ $K_{\mathrm{SDS} / \mathrm{gas}}$ data correlated with the correct form of the model so that we can compare the gas-to-SDS micellar partition process to the many other gas-to-organic solvent partition correlations that have already been derived. Such comparisons can provide valuable information regarding the chemical similarity of different partitioning systems. For example Abraham and Acree ${ }^{6}$ previously showed through Principal Component Analysis (PCA) of equation coefficients that room-temperature ionic liquid solvents have the same solubilizing selectivity as the polar organic solvents, such as acetonitrile, $N, N$-dimethylformamide, $N$-methylpyrrolidinone, and dimethylsulfoxide. The application of PCA in this manner requires that all considered partitioning processes use the same set of solute descriptors.

Finally we have examined the combination of water-toSDS micelle partition coefficient data and micellar electrokinetic chromatographic (MEKC) retention data measured on a micellar sodium dodecyl sulfate pseudostationary phase. Several research groups ${ }^{7-14}$ have correlated their measured chromatographic retention data with the Abraham model; however, no attempt was made to combine the available partition coefficient data and measured MEKC retention values into a single Abraham model correlation.

\section{EXPERIMENTAL DATA AND COMPUTATION METHODS}

Our search of the published chemical literature $e^{2,5,15-33}$ found water-to-aqueous SDS micellar partition coefficient data for 214 different organic compounds for which solute descriptors are known. Most of the experimental data came from the earlier compilation of Abraham et al., ${ }^{2}$ which tabulated the data as $\log P_{\mathrm{x}, \mathrm{SDS} / \text { water }}$ values. In several recent studies researchers expressed the solute concentrations in molarity and reported either the molarity-based partition coefficient, $P_{\mathrm{c}, \mathrm{SDS} / \text { water }}$, or a binding constant $K_{\mathrm{s}}$. The experimental $P_{\mathrm{c}, \mathrm{SDS} / \text { water }}$ and $K_{\mathrm{s}}$ data were converted into the mole fraction water-to-SDS partition coefficients using conversion expressions given in Sepulveda et al. ${ }^{1}$ The experimental log $P_{\mathrm{x}, \mathrm{SDS} / \text { water }}$ data are listed in Table 1, along with the literature references from the values were taken.

Bel'skii $^{5}$ tabulated the gas-to-aqueous SDS micellar phase partition coefficients, $K_{\mathrm{SDS} / \mathrm{gas}}$, of 104 compounds. Many of the compounds listed in Table 1 are not contained in the Bel'skii database. For compounds not in the Bel'skii base we calculated the $\log K_{\mathrm{SDS} / \mathrm{gas}}$ value using eq 7

$$
\log K_{\mathrm{SDS} / \text { gas }}=\log \left(P_{\mathrm{x}, \mathrm{SDS} / \text { water }} V_{\text {aqueous }} / V_{\text {micelle }}\right)+\log K_{\mathrm{W}}
$$

where $V_{\text {micelle }}$ and $V_{\text {aqueous }}$ refer to the molar volume of the micellar and aqueous phase, respectively, and $K_{\mathrm{W}}$ is the solute's gas-to-water partition coefficient. For consistency we used the same values of $V_{\text {micelle }}=0.25 \mathrm{~L} / \mathrm{mol}$ and $V_{\text {aqueous }}$ $=0.018 \mathrm{~L} / \mathrm{mol}$ that were used in generating the Bel'skii database. The gas-to-water partition coefficients needed in the conversion come from our database of $\log K_{\mathrm{W}}$ values. The experimental gas-to-aqueous SDS micellar partition coefficients of 147 compounds are given in Table 2.

Micellar electrokinetic chromatography provides a convenient experimental method for determining water-tomicelle partition coefficients. The measured retention factor, $k$, is directly related to the water-to-micelle partition coefficient and phase ratio, $\varphi$, through eq 8 .

$$
k=P_{\text {micelle/water }} \varphi
$$


Table 1. Logarithm of the Experimental Mole Fraction Water-to-Aqueous SDS Micellar Partition Coefficients, $\log P_{\mathrm{x}, \mathrm{SDS} / \text { water }}$

\begin{tabular}{|c|c|c|c|c|c|c|c|c|c|c|c|c|c|c|c|}
\hline solute & $\mathbf{E}$ & $S$ & A & $\mathbf{B}^{\circ}$ & V & $\log P$ & ref & solute & $\mathbf{E}$ & $\mathbf{S}$ & A & $\mathbf{B}^{\circ}$ & V & $\log P$ & ref \\
\hline argon & 0.000 & 000 & 0.000 & 0.000 & 0.1900 & 1.64 & 2 & 1,10-decanediol & 0.370 & 0.950 & 0.750 & 0.920 & 1.6350 & 3.96 & 2 \\
\hline xygen & 000 & .000 & 0.000 & 0.000 & 0.1830 & 1.64 & 2 & methyl acetate & .142 & 0.640 & 0.000 & 0.450 & 0.6057 & 2.67 & 22 \\
\hline ethane & 0.000 & 0.000 & 0.000 & 0.000 & 0.2495 & 1.82 & 2 & ethyl acetate & .106 & 0.620 & 0.000 & & 0.7466 & 2.72 & 22 \\
\hline hane & 0.000 & 0.000 & 0.000 & 000 & 0.3904 & 2.52 & 2 & propyl acetate & 092 & 0.600 & 0.000 & 0.450 & 0.8875 & 2.78 & 22 \\
\hline & 0.000 & 0.000 & 0.000 & 0.000 & 0.5313 & 3.10 & 2 & tributylphosphine & .270 & 1.160 & 0.000 & 1.660 & 2.0627 & 3.75 & 0 \\
\hline tan & 0.000 & 0.000 & 0.000 & 0.000 & 0.6722 & 3.78 & 2 & benzene & 0.610 & 0.520 & 0.000 & 0.140 & 0.7164 & 2.95 & 2 \\
\hline pentane & 0.000 & 0.000 & 0.000 & 0.000 & 0.8131 & 4.22 & 2 & toluene & 0.601 & 0.520 & 0.000 & 0.140 & 0.8573 & 3.45 & 2 \\
\hline xane & 0.000 & 0.000 & 0.000 & 0.000 & 0.9540 & 4.38 & 2 & ethylbenzen & .613 & 0.510 & 0.000 & 0.150 & 0.9 & 3.98 & 24 \\
\hline etane & 0.000 & 0.000 & 0.000 & 0.000 & 1.0949 & $5.73^{a}$ & 5 & methylber & 613 & 0.520 & 0.000 & 0.160 & 0.9982 & .17 & 0 \\
\hline octane & 0.000 & 0.000 & 0.000 & 0.000 & 1.2358 & $6.41^{a}$ & 5 & & .360 & 0.990 & 0.000 & 0.260 & 1.3240 & 4.83 & \\
\hline nonane & 0.000 & 0.000 & 0.000 & 0.000 & 1.3767 & $7.01^{a}$ & 5 & lene & .340 & 0.920 & 0.000 & 0.200 & 1.0854 & 4.19 & 25 \\
\hline ecane & 0.000 & 0.000 & 0.000 & 0.000 & 1.5176 & $7.57^{a}$ & 5 & ylnaphthale & .344 & 0.900 & 0.000 & 0.200 & 1.2260 & 4.82 & 2 \\
\hline clohe & 0.305 & 0.100 & 0.000 & 0.000 & 0.8454 & 3.55 & 2 & & 2.290 & 1.340 & 0.000 & & & 5.57 & 2 \\
\hline hex & 0.260 & 0.100 & 0.000 & 0.000 & 1.1272 & 4.66 & 16 & $\mathrm{p}$ & & 1.290 & 0.000 & 0.260 & 1.4540 & .31 & 26 \\
\hline ane & -0.580 & 0.260 & 0.000 & 0.000 & 0.3203 & 2.12 & 2 & & 808 & 1.710 & 0.000 & 0.280 & 1.5846 & .91 & 26 \\
\hline chloromethane & 0.387 & 0.570 & 0.100 & 0.050 & 0.4943 & 2.29 & 2 & & .256 & 1.760 & 0.000 & 0.400 & 1.9540 & 7.09 & 26 \\
\hline & 0.425 & 0.490 & 0.150 & 0.020 & 0.6167 & 2.80 & 2 & & .849 & 0.650 & 0.000 & 0.160 & 0.9550 & 3.52 & 16 \\
\hline cortbr & 0.458 & 0.380 & 0.000 & 0.000 & 0.7391 & 3.44 & 2 & lats & .718 & 0.650 & 0.000 & 0.070 & 0.8388 & 3.64 & 15 \\
\hline & 0.436 & 0.610 & 0.110 & 0.050 & & 3.27 & 17 & & & 0.570 & & & & .40 & 15 \\
\hline & 0.425 & 0.410 & 0.090 & 0.050 & 0.5922 & 3.10 & 17 & & 882 & 0.730 & 0.000 & 0.090 & 0.8914 & .04 & 15 \\
\hline & 0.524 & 0.400 & 0.080 & .030 & 0.7150 & 3.44 & 18 & & 188 & 0.820 & 0.000 & 0.120 & & .27 & 15 \\
\hline & 0.639 & 0.440 & 0.000 & 0.000 & 0.8370 & 4.06 & 18 & ne & 72 & 0.780 & & 0.040 & & 3.89 & 2 \\
\hline & 0.640 & 0.400 & 0.000 & 0.150 & 0.6490 & 2.95 & 15 & oro & .847 & 0.730 & 0.000 & 0.020 & 0.9612 & 4.46 & 15 \\
\hline 1. & & & 0.000 & 0 & & & 15 & & & & & & & 60 & 15 \\
\hline bo $>$ & 0.102 & 0.380 & 0.150 & 050 & 10 & 2.78 & 15 & & & & & & & 29 & \\
\hline & 0.329 & 0.750 & 0.000 & 0.640 & & 1.74 & 2 & & 710 & 0.750 & & & & 3.20 & 15 \\
\hline & 0.179 & 0.700 & 0.040 & 0.490 & 0 & 1.53 & 2 & & .871 & 1.110 & & & & 2.98 & 15 \\
\hline none & 0.166 & 0.700 & 0.000 & 0.510 & 0.6879 & 2.11 & 2 & ile & .742 & 1.110 & 0.000 & 0.330 & 0.8710 & 2.80 & 15 \\
\hline & 0.143 & 0.680 & 0.000 & 0.510 & & 2.66 & 32 & & & 1.000 & & & & 2.64 & 15 \\
\hline & 154 & 660 & 0.000 & 10 & 38 & 2.43 & 2 & & & & & & & .34 & 2 \\
\hline & 136 & 0.680 & 0.000 & 0.510 & 0.9697 & 2.93 & 32 & & 916 & 1.580 & 0.000 & 0.530 & & .50 & 2 \\
\hline & 111 & 0.650 & 0.000 & 0.510 & & & 2 & & & 0.950 & & & & 0 & \\
\hline 2-he & 123 & 0.680 & 0.000 & 0.510 & 6 & 3.46 & 2 & & 77 & 0.920 & 0.000 & 0.500 & 1.2 & 3.90 & 2 \\
\hline 2 -oct & 108 & 0.680 & 0.000 & 0.510 & 1.2515 & 3.57 & 15 & none & 1.447 & 1.500 & 0.000 & 0.500 & 1.4810 & 4.46 & 2 \\
\hline & & 30 & 00 & & & & 15 & & & & & & & 2 & 2 \\
\hline & 8 & 680 & 0.000 & 0.510 & & 4. & 15 & & & 0. & & 0.5 & & 4 & 2 \\
\hline & & 860 & 0.000 & 560 & & & 32 & & & 1.520 & & & & 4 & \\
\hline & 188 & .900 & 0.000 & & 50 & 2.38 & 2 & & & 2.450 & 0.400 & 0.800 & & 4.09 & 2 \\
\hline & 0.440 & 1.300 & 0.550 & 0.680 & 0.6468 & $2.24^{a}$ & 2 & & 0.900 & 1.370 & 0.480 & 0.670 & 1.1137 & 3.11 & 2 \\
\hline & 420 & 1.300 & 0.560 & 0.680 & 0.7877 & 2.25 & 2 & & 30 & 0.900 & & & & 3.23 & 2 \\
\hline & & & 00 & 0 & & & 2 & & & & & & & 6 & 2 \\
\hline & 278 & 440 & 0.430 & 0.470 & 2 & 1.2 & 2 & & 30 & 0.900 & 0. & 0. & 26 & 64 & 2 \\
\hline & 246 & 420 & 0.370 & 0.480 & & 1. & 2 & & & 0.900 & & 0.370 & & 4.40 & 2 \\
\hline & 236 & .420 & 0.370 & 0.480 & 0.5900 & 2.0 & 2 & & 0.600 & 0.890 & 0.640 & 0.270 & 0.9414 & 3.41 & 2 \\
\hline & 212 & 360 & 0.330 & 0.560 & 0.5900 & 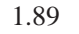 & 2 & & & 0.910 & & 0.290 & 0.9414 & 3.49 & 2 \\
\hline & 24 & 420 & 0.370 & 0 & 99 & & 2 & & & & & & & 3.84 & 2 \\
\hline & & & 70 & 0 & & & 2 & & & 1. & & 0. & & 4 & 2 \\
\hline & & 50 & 0.330 & 0.560 & & 2. & 2 & & & 1.100 & 0.6 & 0.270 & & 3.96 & 2 \\
\hline & & 00 & 0.310 & 0.600 & 99 & & 2 & & & 1.070 & 0.630 & 0.260 & & 3.98 & 2 \\
\hline 1-pe & 219 & 420 & 0.370 & 0.480 & 0.8718 & 2.91 & 2 & & 1.310 & 1.130 & 0.660 & 0.310 & & 4.13 & 2 \\
\hline & & & 0.330 & & & 2.69 & 2 & & & & & & & 4.17 & 2 \\
\hline & & & & & & & 2 & & & & & & & 3.26 & 30 \\
\hline & 2 & 0 & 0.370 & 480 & 18 & 2.88 & 2 & & 10 & 0.8 & 0.8 & 0.580 & 04 & 3.22 & 2 \\
\hline & & & & 30 & & 3.00 & 19 & & & & & & & 2.54 & 2 \\
\hline 2-me & 0.194 & 300 & 0.310 & 0.600 & 0.8718 & $2.6^{\circ}$ & 2,1 & & & 0.890 & 0.580 & 0.580 & 1.1313 & 3.51 & 2 \\
\hline $2,2-$ & 0.220 & 360 & 0.370 & & & 2.63 & 2 & & & & 0.620 & 0.520 & & 3.49 & 2 \\
\hline & & & & & & & 2 & & & & & & & 3.31 & 2 \\
\hline & & 0 & 0.330 & 0 & & 3. & 2 & & & & & 0.5 & & 3.40 & 2 \\
\hline & & & & & & 3. & 21 & & & & & & & 3.45 & \\
\hline $4-n$ & & 390 & 0.370 & 0.480 & 1.0127 & 3.39 & 19 & & & 1.520 & 0.680 & 0.400 & 1.1059 & 3.49 & 2 \\
\hline $4-m$ & 0.167 & 330 & 0.330 & 0.560 & 1.0127 & 3.35 & 19 & & .940 & 1.310 & 0.650 & 0.690 & 1.2292 & 3.52 & 2 \\
\hline anol & & & 0.330 & & & 3.26 & 19 & & & & & 0.670 & & 3.49 & 2 \\
\hline & & & & & & & 2 & & & & & & & 3.56 & 30 \\
\hline & & & & & & 3.7 & 2 & & & & & 0.3 & & 296 & \\
\hline -1-nexanor & & & & & & 3.81 & 19 & & & & & 0.300 & & 3.08 & 2 \\
\hline $1-0$ & 0.199 & 420 & 0.370 & 0.480 & 1.2950 & 4.22 & 2 & & 0.822 & 0.880 & 0.570 & 0.340 & 0.9160 & 3.14 & 2 \\
\hline 6-methyl-1-heptanol & 0.216 & 390 & 0.370 & 0.480 & 1.2950 & 4.21 & 19 & phenol & 0.820 & 0.870 & 0.570 & 0.310 & 0.9160 & 3.14 & 2 \\
\hline & 191 & 420 & 0.370 & 0.480 & & $4.41^{a}$ & 2 & & & 0.820 & & 0.370 & & 3.52 & \\
\hline & & & & & & 1. & 2 & & & & & & & 3.56 & \\
\hline & & & & & & & 2 & & & & & 0.3 & & 3.56 & \\
\hline & & & & 0 & & 1.45 & 2 & & & 0.820 & & & & 3.52 & 2 \\
\hline 1,3-butanediol & 0.377 & .900 & 0.700 & 0.830 & 0.7896 & 1.52 & 23 & hylphenol & 0.830 & 0.860 & 0.550 & 0.370 & 1.0569 & 3.56 & 2 \\
\hline 1,4-butanediol & 0.395 & 0.930 & 0.720 & 0.900 & 0.7896 & 1.65 & 2 & 2,4,5-trimethylphen & 0.850 & 0.790 & 0.520 & 0.440 & 1.1980 & 3.85 & 2 \\
\hline & & 0.930 & 0.610 & 0.880 & 0.7896 & 1.54 & 23 & tramethylphe & 0.880 & 0.810 & 0.380 & 0.440 & & 4.19 & \\
\hline 1,6-hex & 0.385 & 0.950 & 0.750 & 0.920 & 1.0714 & 2.38 & 2 & 4-ethylphenol & 0.800 & 0.900 & 0.550 & 0.360 & 1.0569 & 3.60 & \\
\hline
\end{tabular}


Table 1 (Continued)

\begin{tabular}{|c|c|c|c|c|c|c|c|c|c|c|c|c|c|c|c|}
\hline solute & $\mathbf{E}$ & $\mathbf{S}$ & $\mathbf{A}$ & $\mathbf{B}^{\circ}$ & $\mathbf{V}$ & $\log P$ & ref & solute & $\mathbf{E}$ & $\mathbf{S}$ & $\mathbf{A}$ & $\mathbf{B}^{\circ}$ & $\mathbf{V}$ & $\operatorname{gg} P$ & ref \\
\hline 4-propylphenol & 0.793 & 0.880 & 0.550 & 0.370 & 1980 & 4.18 & 2 & 18-crown-6 & 0.400 & 1.340 & 0.000 & 2.130 & 2.0430 & 3.91 & 2 \\
\hline 4-tert-butylphenol & 310 & 0.890 & 0.560 & 0.390 & 1.3390 & 4.32 & 2 & 15-crown-5 & 0.410 & 1.200 & 0.000 & 1.750 & 1.7025 & 3.19 & 2 \\
\hline 4-tert-pentylphenol & 0.810 & 0.890 & 0.560 & 0.410 & 1.4800 & 4.76 & 2 & 12-crown-4 & 0.420 & 0.990 & 0.000 & 1.390 & 1.3620 & 2.42 & 2 \\
\hline 4-fluorophenol & 0.670 & 0.970 & 0.630 & 0.230 & 0.7930 & 2.99 & 2 & butyl urea & 0.372 & 1.450 & 0.620 & 0.670 & 1.0284 & 2.51 & 2 \\
\hline 4-chlorophenol & 0.909 & 1.060 & 0.690 & 0.150 & 0.8975 & 3.24 & 2 & 2,5-hexanediol & 0.354 & 0.900 & 0.720 & 0.900 & 1.0714 & 2.21 & 2 \\
\hline 4-bromophenol & 080 & 1.170 & 0.670 & 0.200 & 0.9500 & 3.54 & 2 & 4-tert-butoxybenzoi & 0.830 & 0.880 & 0.580 & 0.520 & 1.5540 & 4.65 & 2 \\
\hline 4-iodophenol & 380 & 1.220 & 0.680 & 0.200 & 1.0330 & 3.91 & 2 & pentafluorophenol & 0.360 & 0.830 & 0.790 & 0.090 & 0.8636 & $3.92^{a}$ & 2 \\
\hline 2,4-dichlorophenol & 0.960 & 0.990 & 0.580 & 0.140 & 1.0200 & 4.09 & 31 & allylthiourea & 0.940 & 0.760 & 0.450 & 0.950 & 0.9493 & 2.11 & 2 \\
\hline 2,6-dichlorophenol & 0.900 & 0.900 & 0.380 & 0.240 & 1.0200 & 3.92 & 31 & 4-bromo-p-terphenyl & 2.310 & 1.690 & 0.000 & 0.250 & 2.1070 & 6.74 & 2 \\
\hline 3,5-dichlorophenol & 1.020 & 1.000 & 0.910 & 0.000 & 1.0200 & 3.72 & 2 & 3-phenoxy-1-propanol & 0.850 & 0.950 & 0.310 & 0.780 & 1.2565 & 3.38 & 2 \\
\hline 2,3,5-trichlorophenol & 1.070 & 0.940 & 0.680 & 0.160 & 1.1420 & 4.20 & 2 & 2,4,6-tribromophenol & 1.620 & 1.340 & 0.500 & 0.160 & 1.3001 & 5.07 & 2 \\
\hline nol & 1.010 & 1.010 & 0.820 & 0.080 & 1.1420 & 4.39 & 31 & 2,4 & 58 & 1.540 & 0.300 & 0.200 & 97 & .11 & 2 \\
\hline henol & 1.170 & 0.880 & 0.700 & 0.130 & 1.2650 & 4.68 & 2 & n-trif & 1.080 & 1.030 & 0.000 & 0.340 & 1.2970 & 4.37 & 2 \\
\hline pentachlorophens & 1.217 & 0.860 & 0.610 & 0.090 & 1.3870 & 5.13 & 2 & ferro & 1.350 & 0.850 & 0.000 & 0.200 & 1.1209 & 4.28 & 2 \\
\hline 3-(trifluoromethyl)phenol & 0.425 & 0.870 & 0.720 & 0.090 & 0.9691 & 3.87 & 2 & acetate & 0.798 & 1.060 & 0.000 & 0.650 & 1.2140 & 3.19 & 27 \\
\hline 4-methoxyphenol & 0.900 & 1.170 & 0.570 & 0.480 & 0.9750 & 2.93 & 2 & 1-nitrobutane & 0.227 & 0.950 & 0.000 & 0.290 & 0.8470 & 3.00 & 15 \\
\hline & 0.870 & 1.170 & 0.570 & 0.520 & 1.1160 & & 2 & & & 0.950 & 0.000 & 0.2 & & 3.41 & 15 \\
\hline enol & 1.010 & 1.540 & 0.850 & 0.370 & 0.9320 & 2.85 & 2 & $1-1$ & 0.203 & 0.950 & 0.000 & 0.290 & 1.1290 & 3.88 & 15 \\
\hline 4-cy & 0.940 & 1.630 & 0.800 & 0.290 & .9300 & 2.93 & 2 & 3, & 1.650 & 1.180 & 0.000 & 0.160 & 1.5690 & 5.82 & 28 \\
\hline 4-nitr & 1.070 & 1.720 & 0.820 & 0.260 & 0.9493 & 2.96 & 2 & 4,4'-dichlor & 1.640 & 1.180 & 0.000 & 0.160 & 1.5690 & 6.20 & 28 \\
\hline catechol & 0.970 & 1.100 & 0.880 & 0.470 & 0.8338 & 2.56 & 2 & 2,4,6-trichlorobi & 1.740 & 1.350 & 0.000 & 0.170 & 1.6914 & 6.43 & 28 \\
\hline methyl 4-hydroxy- & 0.900 & 1.370 & 0.690 & 0.450 & 1.1313 & 3.33 & 2 & $2,4,5$ & 1.770 & 1.330 & 0.000 & 0.150 & 1.6914 & 6.44 & 28 \\
\hline & & & & & & & & $2,2^{\prime}$ & 1.900 & 1.480 & 0.000 & 0.150 & 1.8138 & 6.60 & 28 \\
\hline ethyl 4-hydroxybenzoate & 0.860 & 1.350 & 0.690 & 0.450 & 1.2722 & 3.60 & 2 & biphenvl & & & & & & & \\
\hline $\begin{array}{l}\text { propyl 4-hydroxy- } \\
\text { benzoate }\end{array}$ & 0.860 & 1.350 & 0.690 & 0.450 & 1.4131 & 3.99 & 2 & $\begin{array}{l}\text { 2,3,4,5-tetrachloro- } \\
\text { biphenyl }\end{array}$ & 1.920 & 1.460 & 0.000 & 0.130 & 1.8138 & 6.86 & 28 \\
\hline $\begin{array}{l}\text { methyl 2-hydroxy- } \\
\text { benzoate }\end{array}$ & 0.850 & 840 & 0.040 & 0.460 & 1.1313 & 3.60 & 29 & $\begin{array}{l}\text { 2,3,4,5,6-pentachloro- } \\
\text { biphenyl }\end{array}$ & 40 & 1.610 & 0.000 & 30 & 52 & 6.99 & 28 \\
\hline nanhthol & 520 & 1.050 & 0.600 & 0.370 & 1440 & 4.0 & 2 & $2,2^{\prime}, 3,3^{\prime}, 6,6^{\prime}$-hexachloro- & 2.140 & 1.740 & 0.000 & 0.110 & 2.0586 & 7.13 & 28 \\
\hline & 303 & 0.870 & 0.390 & 0.560 & 9160 & & 2 & & & & & & & & \\
\hline 2-phenyl-1-ethanol & 0.811 & 0.860 & 0.310 & 0.650 & 1.0569 & 3.12 & 2 & $2,2^{\prime}, 3,3^{\prime}, 4,4^{\prime}$-hexachloro- & 2.180 & 1.740 & 0.000 & 0.110 & 2.0586 & 7.53 & 28 \\
\hline 3-phenyl-1-propanol & 0.821 & 0.940 & 0.310 & 0.650 & 1.1978 & 3.40 & 2 & & & & & & & & \\
\hline 4-phenyl-1-butanol & 0.811 & 0.900 & 0.330 & 0.700 & 1.3390 & 3.77 & 2 & methyl methacrylate & 0.245 & 0.510 & 0.000 & 0.440 & 0.8445 & 2.85 & 20 \\
\hline & 0.804 & 0.900 & 0.330 & 0.720 & 1.4800 & 4.19 & 2 & & 0.149 & 0.570 & 0.000 & 0.450 & 1.2680 & 4.10 & 20 \\
\hline & 0.804 & 0.900 & 0.330 & 0.720 & 1.6200 & 4.5 & 2 & & & 1.340 & 0.000 & 0.380 & 1.0902 & 3.35 & 33 \\
\hline 2-phenoxyethanol & 0.850 & 0.950 & 0.300 & 0.780 & 1.1156 & 3.11 & 2 & 4-methoxyanisole & 0.970 & 1.290 & 0.000 & 0.400 & 1.0902 & 3.34 & 33 \\
\hline
\end{tabular}

The phase ratio is defined as the volume of the micellar pseudo-stationary phase over that of the bulk aqueous phase ( $\left.v_{\text {micelle }} / v_{\text {aqueous }}\right)$ and can be calculated from

$$
\varphi=\frac{v_{\text {micelle }}}{v_{\text {aqueous }}}=\frac{v\left(C_{\text {surf }}-\mathrm{cmc}\right)}{1-v\left(C_{\text {surf }}-\mathrm{cmc}\right)}
$$

a knowledge of the surfactant's partial specific molar volume, $v$, the critical micelle concentration, $\mathrm{cmc}$, and the surfactant's molar concentration, $C_{\text {surf. }}$. Our search of the chemical literature found 8 sets of experimental SDS micellar MEKC retention factor data. The 259 numerical values are reported in Table S1 (Supporting Information), along with the literature reference to indicate where each set of values was taken. Several solutes are listed multiple times in Table S1 as we felt that it would be inappropriate to average retention factors that were measured under different experimental conditions.

Molecular descriptors for all of the compounds considered in the present study are also tabulated in Tables 1, 2, and $\mathrm{S} 1$. The numerical values came from our solute descriptor database, which now contains values for more than 4000 different organic and organometallic compounds. The descriptors were obtained exactly as described before, using various types of experimental data, including water to solvent partitions, gas to solvent partitions, solubility, and chromatographic data. ${ }^{34}$ Solute descriptors used in the present study are all based on experimental data. There is also commercial software $^{35}$ and several published estimation schemes ${ }^{36-39}$ available for calculating the numerical values of solute descriptors from molecular structural information if one is unable to find the necessary partition, solubility, and/or chromatographic data.

\section{RESULTS AND DISCUSSION}

We have assembled in Table 1 values of $\log P_{\mathrm{x}, \mathrm{SDS} / \text { water }}$ for 214 solutes covering a reasonably wide range of compound type and descriptor values. Preliminary analysis of the experimental data in accordance with the Abraham model identified propionamide, 1-decanol, pentafluorophenol, heptane, octane, nonane, and decane as outliers. The large deviations noted in the case of the four alkane solutes were expected as Treiner and Manneback ${ }^{40}$ had suggested previously that alkane solutes behave differently than other solutes in that alkanes are sorbed by the hydrophobic alkyl chain of the surfactant micelle. There is nothing structurally unusual about the other three solutes to suggest why they should exhibit anything other than normal partition behavior, and a likely reason for why the molecules were identified as outliers is possible experimental error. In an experimental data set containing more than 200 compounds a few outliers are to be expected. The seven compounds were removed from the database, and the final regression was performed to yield the following correlation equation: 
Table 2. Logarithm of the Experimental Gas-to-Aqueous SDS Micellar Partition Coefficients, $\log K_{\mathrm{SDS} / \mathrm{gas}}$

\begin{tabular}{|c|c|c|c|c|c|c|c|c|c|c|c|c|c|c|c|}
\hline solute & $\mathbf{E}$ & $\mathbf{S}$ & $\mathbf{A}$ & $\mathbf{B}^{\circ}$ & $\mathbf{L}$ & $\log K$ & ref & solute & $\mathbf{E}$ & $\mathbf{S}$ & $\mathbf{A}$ & $\mathbf{B}^{\circ}$ & $\mathbf{L}$ & $\log K$ & ref \\
\hline argon & 0.000 & 0.000 & 0.000 & 0.000 & -0.688 & -1.002 & 5 & butylbenzene & 0.600 & 0.510 & 0.000 & 0.150 & 4.730 & 3.918 & 5 \\
\hline oxygen & 0.000 & 0.000 & 0.000 & 0.000 & -0.723 & -1.057 & 5 & pentylbenzene & 0.594 & 0.510 & 0.000 & 0.150 & 5.23 & 4.130 & 5 \\
\hline methane & 0.000 & 0.000 & 0.000 & 0.000 & -0.323 & -0.780 & 5 & 1,4-dimethylbenzene & 0.613 & 0.520 & 0.000 & 0.160 & 3.839 & 3.617 & 5 \\
\hline ethane & 0.000 & 0.000 & 0.000 & 0.000 & 0.492 & 0.049 & 5 & biphenyl & 1.360 & 0.990 & 0.000 & 0.260 & 6.014 & 5.649 & \\
\hline propane & 0.000 & 0.000 & 0.000 & 0.000 & 1.050 & 0.523 & 5 & naphthalene & 1.340 & 0.920 & 0.000 & 0.200 & 5.161 & 4.928 & \\
\hline butane & 0.000 & 0.000 & 0.000 & 0.000 & 1.615 & 1.124 & 5 & 1-methylnaphthalene & 1.344 & 0.900 & 0.000 & 0.200 & 5.802 & 5.456 & $\tilde{E}$ \\
\hline pentane & 0.000 & 0.000 & 0.000 & 0.000 & 2.162 & 1.620 & 5 & anthracene & 2.290 & 1.340 & 0.000 & 0.280 & 7.568 & 7.327 & 5 \\
\hline hexane & 0.000 & 0.000 & 0.000 & 0.000 & 2.668 & 1.906 & 5 & phenanthrene & 2.055 & 1.290 & 0.000 & 0.260 & 7.632 & 7.307 & 5 \\
\hline heptane & 0.000 & 0.000 & 0.000 & 0.000 & 3.173 & $2.624^{a}$ & 5 & pyrene & 2.808 & 1.710 & 0.000 & 0.280 & 8.833 & 8.377 & 5 \\
\hline octane & 0.000 & 0.000 & 0.000 & 0.000 & 3.677 & $3.160^{a}$ & 5 & perylene & 3.256 & 1.760 & 0.000 & 0.400 & 12.053 & 11.687 & 26 \\
\hline nonane & 0.000 & 0.000 & 0.000 & 0.000 & 4.182 & $3.565^{a}$ & 5 & styrene & 0.849 & 0.650 & 0.000 & 0.160 & 3.856 & 3.291 & 16 \\
\hline decane & 0.000 & 0.000 & 0.000 & 0.000 & 4.686 & $4.105^{a}$ & 5 & chlorobenzene & 0.718 & 0.650 & 0.000 & 0.070 & 3.657 & 3.373 & 5 \\
\hline cyclohexane & 0.305 & 0.100 & 0.000 & 0.000 & 2.964 & 1.507 & 2 & fluorobenzene & 0.477 & 0.570 & 0.000 & 0.100 & 2.788 & 3.091 & 5 \\
\hline ethylcyclohexane & 0.260 & 0.100 & 0.000 & 0.000 & 3.877 & 1.937 & 16 & bromobenzene & 0.882 & 0.730 & 0.000 & 0.090 & 4.041 & 3.974 & 5 \\
\hline tetrafluoromethane & -0.580 & -0.260 & 0.000 & 0.000 & -0.817 & -1.313 & 2 & iodobenzene & 1.188 & 0.820 & 0.000 & 0.120 & 4.502 & 4.412 & 5 \\
\hline dichlorometl & 0.387 & 0.570 & 0.100 & 0.050 & 2.019 & 2.107 & 5 & 1,2 & 0.872 & 0.780 & 0.000 & 0.040 & 4.518 & 4.271 & 5 \\
\hline chloroform & 0.425 & 0.490 & 0.150 & 0.020 & 2.480 & 447 & 5 & hlorob & 0.847 & 0.730 & 0.000 & 0.020 & 4.410 & 4.044 & \\
\hline carbon tetrachloride & 0.458 & 0.380 & 0.000 & 0.000 & 2.823 & 2.237 & 5 & blorohen & 0.825 & 0.750 & 0.000 & 0.020 & 4.435 & 4.193 & 15 \\
\hline cis-dichlo & 0.436 & 0.610 & 0.110 & 0.050 & 2.439 & 2.987 & 17 & anisole & 0.710 & 0.750 & 0.000 & 0.290 & 3.890 & 3.864 & 5 \\
\hline trans-dichloroethylene & 0.425 & 0.410 & 0.090 & 0.050 & 2.278 & 2.527 & 17 & nitrobenzene & 0.871 & 1.110 & 0.000 & 0.280 & 4.557 & 4.964 & 5 \\
\hline trich & 0.524 & 0.400 & 0.080 & 0.030 & 2.997 & 2.621 & 18 & & 0.742 & 1.110 & 0.000 & 0.330 & 4.039 & 5.211 & 5 \\
\hline tetrachloro & 0.639 & 0.440 & 0.000 & 0.000 & 3.584 & 849 & 18 & ben & 820 & 1.000 & 0.000 & 0.390 & 4.008 & 4.448 & 15 \\
\hline iodoethane & 0.640 & 0.400 & 0.000 & 0.150 & 2.573 & 2.484 & 5 & enone & 0.818 & 1.010 & 0.000 & 0.480 & 4.501 & 5.557 & 5 \\
\hline 1-iodobutan & 0.628 & 0.400 & 0.000 & 0.150 & 3.6280 & 2.839 & 5 & $4-1$ & 0.916 & 1.580 & 0.000 & 0.530 & 5.935 & 7.397 & 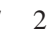 \\
\hline halothane & 0.102 & 0.380 & 0.150 & 0.050 & 2.177 & 1.723 & 5 & be & 1.447 & 1.500 & 0.000 & 0.500 & 6.852 & 8.117 & 2 \\
\hline 1,4-dioxane & 0.329 & 0.750 & 0.000 & 0.640 & 2.892 & 4.307 & 5 & aniline & 0.955 & 0.960 & 0.260 & 0.500 & 3.934 & 5.807 & 5 \\
\hline tone & 0.179 & 0.700 & 0.040 & 0.490 & 1.696 & 77 & 5 & 4 & 0.923 & 0.950 & 0.230 & 0.520 & 52 & 87 & 5 \\
\hline $2-b$ & 0.166 & 0.700 & 0.000 & 0.510 & 2. & 41 & 5 & & 00 & 1.370 & 0.480 & 0.670 & & 777 & 2 \\
\hline 2-pentanone & 0.143 & 0.680 & 0.000 & 0.510 & 2.755 & 4.14 & 5 & be & 0.730 & 0.900 & 0.590 & 0.400 & 4.510 & 7.187 & 2 \\
\hline & 0.154 & 0.660 & 0.000 & 0.510 & & & 5 & & 0.730 & 0.890 & 0.600 & 0.400 & 19 & 497 & 2 \\
\hline 2-hexanone & 136 & 0.680 & 0.000 & 0.510 & 86 & 37 & 5 & cid & 0.730 & 0.900 & 0.600 & 0.430 & 4. & 7.517 & 2 \\
\hline 4-methyl-2-pentanone & 0.111 & 0.650 & 0.000 & 0.510 & 3.089 & 3.947 & 5 & 3-chlorobenzoic acid & 0.840 & 0.950 & 0.630 & 0.320 & 5.197 & 7.847 & 2 \\
\hline none & 0.123 & 0.680 & 0.000 & 0.510 & & & 5 & c acid & 0.840 & 1.020 & 0.630 & 0.270 & 47 & 7.497 & 2 \\
\hline & 108 & 0.680 & 0.000 & 0.510 & & & 5 & & & & 0.6 & 0.270 & & 87 & 2 \\
\hline 2-nonanone & 0.119 & 0.680 & 0.000 & 0.510 & 4.735 & 5.164 & 5 & & 1.310 & 1.150 & 0.640 & 0.310 & 6.351 & 8.627 & 2 \\
\hline & 108 & 0.680 & 0.000 & 0.510 & 5.245 & 4 & 5 & & 0.910 & 0.880 & 0.860 & 0.580 & 4.860 & 9.077 & 2 \\
\hline acetor & 0.237 & 0.900 & 0.070 & 0.320 & 1.739 & 3.087 & 5 & $c$ acid & 0.930 & 0.900 & 0.810 & 0.560 & 4.867 & 8.177 & 2 \\
\hline propanenitrile & 0.162 & 0.900 & 0.020 & 0.360 & 2.082 & 3.507 & 5 & 4-methoxybenzoic acid & 0.899 & 1.250 & 0.620 & 0.520 & 5.741 & 9.047 & 2 \\
\hline bu & 188 & 0.900 & 0.000 & 0.360 & & 37 & 5 & $3-1$ & 0.990 & & 0.730 & 0.520 & 1 & 37 & 2 \\
\hline trile & 177 & 0.900 & 0.000 & 0.360 & & 247 & 5 & $4-$ & 90 & 1.5 & 0.6 & 0.400 & & 247 & 2 \\
\hline & 0.278 & 0.440 & 0.430 & 0.470 & 0.970 & 819 & 5 & phe & 0.805 & 0.890 & 0.600 & 0.300 & 3.766 & 6.464 & 5 \\
\hline & 246 & 0.420 & 0.370 & 0.480 & & & 5 & & 0.840 & 0.860 & 0.520 & 0.300 & 4.218 & 6.247 & 5 \\
\hline 1-propanol & 236 & 0.420 & 0.370 & 0.480 & 2.031 & 4.441 & 5 & $3-1$ & 0.822 & 0.880 & 0.570 & 0.340 & 4.31 & 6.597 & 2 \\
\hline 2-propanol & 0.212 & 0.360 & 0.330 & 0.560 & 1.764 & 4.227 & 5 & & 0.820 & 0.870 & 0.570 & 0.310 & 4.312 & 6.497 & 5 \\
\hline 1-butanol & 224 & 0.420 & 0.370 & 0.480 & 2.601 & 22 & 5 & 2, & 0.850 & & & 0.370 & 95 & 6.897 & 5 \\
\hline nol & 17 & 00 & 0 & 0 & & & 5 & & 3 & 0. & 0. & 0.400 & & 27 & 5 \\
\hline atanol & 0.217 & 0.360 & 0.330 & 0.560 & 2.338 & 657 & 5 & & 0.840 & 0.830 & 0.500 & 0.380 & 4.774 & 6.757 & 5 \\
\hline & 0.180 & 0.300 & 0.310 & 0.600 & & & 5 & & 0.850 & 0.820 & 0.510 & 0.370 & 4.680 & 6.237 & 5 \\
\hline 1-pentanol & 0.219 & 0.420 & 0.370 & 0.480 & 3.106 & 5.185 & 5 & $3,5-c$ & 0.830 & 0.860 & 0.550 & 0.370 & 4.856 & 7.017 & 5 \\
\hline 2-pentanol & 0.195 & 0.360 & 0.330 & 0.560 & 2.840 & 4.772 & 5 & & 0.800 & 0.900 & 0.550 & 0.360 & 4.737 & 6.96 & 5 \\
\hline & 218 & 0.360 & 0.330 & & & & 5 & & 0.793 & 0.880 & 0.5 & 0.370 & & 7.367 & 5 \\
\hline $3-\mathrm{m}$ & 192 & 0.390 & 370 & 0.480 & 30 & 4.977 & 5 & & 0.810 & 0.890 & 0.560 & 0.390 & 54 & 7.517 & 5 \\
\hline & 194 & 0.330 & 0.330 & 0.580 & 2.7930 & 5.007 & 19 & $4-1$ & 0.670 & 0.970 & 0.630 & 0.230 & 3.844 & 6.387 & 5 \\
\hline $2-\mathrm{m}$ & 194 & 0.300 & 0.310 & 0.600 & 2.630 & 4.697 & 5 & & 0.909 & 1.060 & 0.690 & 0.150 & 4.775 & 7.257 & 5 \\
\hline 1-hexanol & 0.210 & 0.420 & 0.370 & 0.480 & 3.610 & 5.551 & 5 & 4-bromophenol & 1.080 & 1.170 & 0.670 & 0.200 & 5.135 & 7.627 & 5 \\
\hline 2-hexanol & 0.187 & 0.360 & 0.330 & 0.560 & 3.340 & 5.067 & 5 & pentachlorophen & 1.217 & 0.860 & 0.610 & 0.090 & 6.864 & 7.727 & 2 \\
\hline 3-hexanol & 200 & 0.360 & 0.330 & 0.560 & 3.343 & 4.867 & 21 & 4-methoxyphenol & 0.900 & 1.170 & 0.570 & 0.480 & 4.773 & 7.407 & 2 \\
\hline $4-n$ & 67 & 0.330 & 0.330 & 0. & & & 19 & & 0.940 & 1.630 & 0.800 & 0.290 & 20 & 9.247 & 5 \\
\hline $2-$ & 07 & 0.330 & 0.330 & 0.560 & 3.240 & 050 & 19 & $4-n$ & 1.070 & 1.720 & 0.820 & 0.260 & 76 & 9.627 & 5 \\
\hline 1-heptanol & 0.211 & 0.420 & 0.370 & 0.480 & 4.115 & 5.687 & 5 & catec & 0.970 & 1.100 & 0.880 & 0.470 & 4.450 & 8.617 & 2 \\
\hline 2-heptanol & 0.188 & 0.360 & 0.330 & 0.560 & 3.842 & 5.197 & 2 & methyl 4-hydroxybenzo & 0.900 & 1.370 & 0.690 & 0.450 & 5.665 & 9.027 & 2 \\
\hline 1-octanol & 0.199 & 0.420 & 0.370 & 0.480 & 4.619 & 6.077 & 5 & 1-naphthol & 1.520 & 1.050 & 0.600 & 0.370 & 6.130 & 8.497 & 5 \\
\hline 1-decanol & .191 & 0.420 & 0.370 & 0.480 & & $5.937^{a}$ & 2 & 2-naphthol & 1.520 & 1.080 & 0.610 & 0.400 & 6.200 & 8.785 & 5 \\
\hline & 237 & 0.520 & 0.310 & 0.810 & 2.792 & .507 & 2 & ben & 0.803 & 0.870 & 0.390 & 0.560 & 4.221 & 6.577 & 5 \\
\hline & 201 & 0.500 & 0.300 & 0.830 & 3.806 & 6.487 & 5 & $2-p$ & 0.811 & 0.860 & 0.310 & 0.650 & 4.628 & 6.957 & 5 \\
\hline 1,3 & 591 & 0.910 & 0.720 & 0.850 & 3.263 & & 2 & -1-propanol & 0.821 & 0.940 & 0.310 & 0.650 & 5.312 & 7.342 & 5 \\
\hline 1,3-butanediol & 0.377 & 0.900 & 0.700 & 0.830 & 3.642 & 7.591 & 23 & 1-nitrobutane & 0.227 & 0.950 & 0.000 & 0.290 & 3.415 & 4.08 & 5 \\
\hline methyl acetate & 0.142 & 0.640 & 0.000 & 0.450 & 1.911 & 3.824 & 22 & 1-nitropentane & 0.212 & 0.950 & 0.000 & 0.290 & 3.938 & 4.298 & 5 \\
\hline ethyl acetate & 0.106 & 0.620 & 0.000 & 0.450 & 2.314 & 3.736 & 22 & $4,4^{\prime}$-dichlorobiphe & 1.640 & 1.180 & 0.000 & 0.160 & 7.580 & 7.184 & 28 \\
\hline propyl acetate & 092 & 0.600 & 0.000 & 0.450 & 2.819 & 3.685 & 22 & ben & 0.990 & 1.500 & 0.490 & 0.670 & 5.767 & 9.553 & 5 \\
\hline & 610 & 0.520 & 0.000 & 0.140 & & 2.644 & 5 & & 0.366 & 0.400 & 0.000 & 0.120 & 2.120 & 2.147 & 5 \\
\hline & & 0.520 & 0.000 & & & & 5 & & 0.366 & 0.400 & 0.000 & 0.120 & & 2.707 & 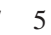 \\
\hline enzene & 0.613 & 0.510 & 0.000 & 0.150 & 3.778 & 3.358 & 5 & 1-bromobutane & 0.340 & 0.400 & 0.000 & 0.120 & 3.1050 & 2.977 & 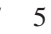 \\
\hline propylbenzene & 0.604 & 0.500 & 0.000 & 0.150 & 4.230 & 3.599 & 5 & & & & & & & & \\
\hline
\end{tabular}




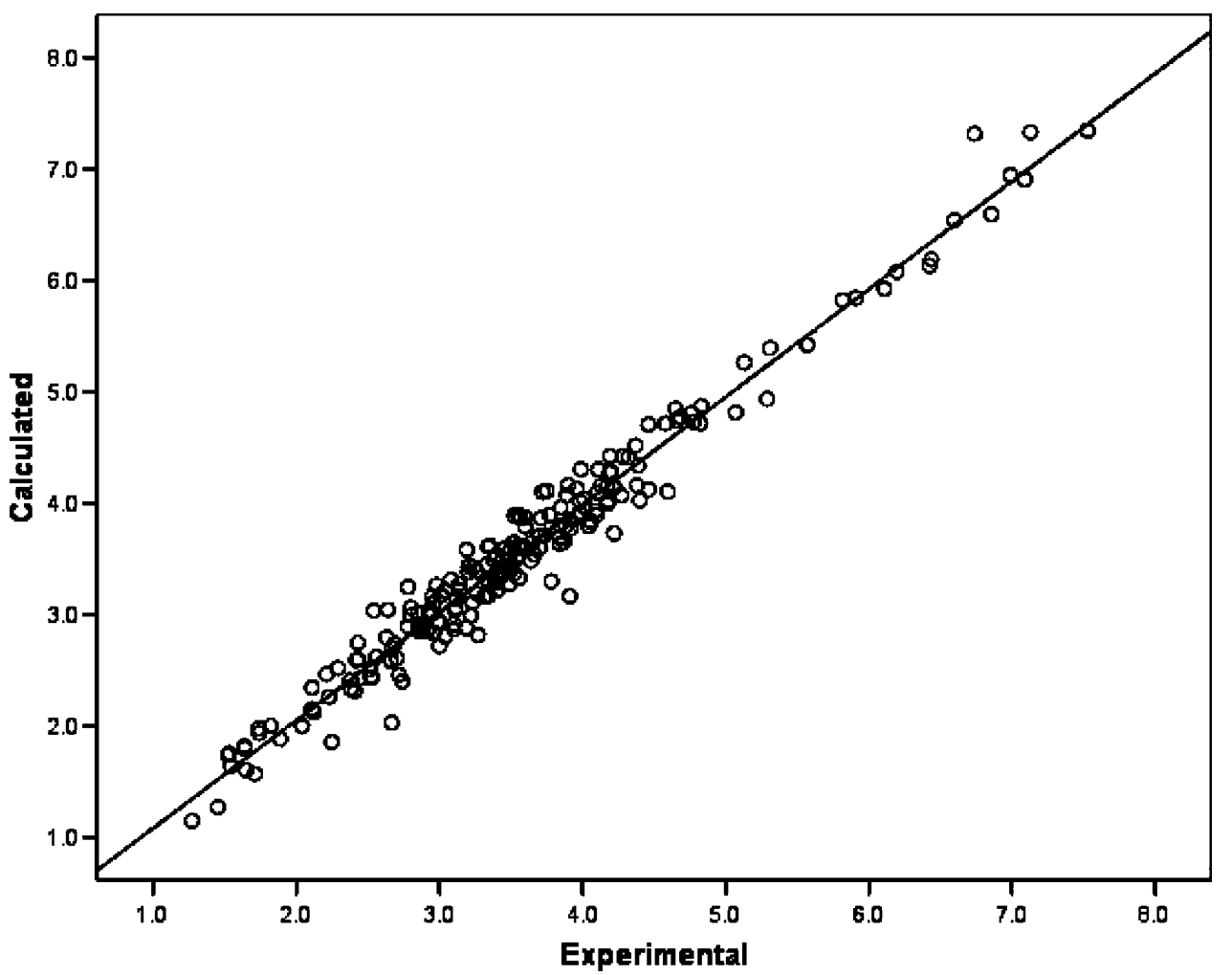

Figure 1. Experimental $\log P_{\mathrm{x}, \mathrm{SDS} / \text { water }}$ vs the calculated values based on eq 10 .

All regression analyses were performed using SPSS statistical software. The equation coefficients of eq 10 are nearly identical to those of the correlation derived previously by Abraham et al. ${ }^{2}$ The coefficients for eq 10 reflect the difference in properties of the SDS micellar phase from those of water. The negative $a$ and $b$ coefficients indicate that the micellar phase is both a weaker hydrogen bond acid and a weaker hydrogen bond base than water as expected. Figure 1 compares the calculated values of $\log P_{\mathrm{x}, \mathrm{SDS} / \text { water }}$ based on eq 10 against the observed values.

In order to assess the predictive ability of eq 10 we divided the 207 data points into a training set and a test set by allowing the SPSS software to randomly select half of experimental values. The selected data points became the training set, and the compounds that were left served as the test set. Analysis of the experimental data in the training set gave

$$
\begin{gathered}
\log P_{\mathrm{x}, \mathrm{SDS} / \text { water }}=1.327(0.067)+0.366(0.064) \mathbf{E}- \\
0.407(0.085) \mathbf{S}-0.126(0.077) \mathbf{A}-1.977(0.091) \mathbf{B}^{\circ}+ \\
2.981(0.087) \mathbf{V}(11) \\
\left(N=104, R^{2}=0.975, \mathrm{SD}=0.189, F=751.17\right)
\end{gathered}
$$

There is very little difference in the equation coefficients for the full data set and training data set correlations. The training set correlation was then used to predict $\log P_{\mathrm{x}, \mathrm{SDS} / \text { water }}$ values for the 103 compounds in the test set. For the

$$
\log P_{\mathrm{x}, \mathrm{SDS} / \text { water }}=1.239(0.052)+0.384(0.052) \mathbf{E}-0.482
$$$$
(0.064) \mathbf{S}-0.190(0.054) \mathbf{A}-1.803(0.065) \mathbf{B}^{\circ}+3.064
$$

$$
\left(N=207, R^{2}=0.968, \mathrm{SD}=0.203, F=1211.58\right)
$$

predicted and experimental values, we find that $\mathrm{SD}=0.2324$, $\mathrm{AAE}$ (average absolute error) $=0.1722$ and the $\mathrm{AE}$ (average error) $=-0.0019$. There is therefore very little bias in the predictions using eq 11 with $\mathrm{AE}$ equal to -0.0019 .

Table 2 contains the 104 gas-to-aqueous SDS micellar partition coefficients, expressed as $\log K_{\mathrm{SDS} / \mathrm{gas}}$, used by Bel'skii in deriving eq 6 plus numerical values for an additional 39 organic compounds that were calculated from experimental $\log P_{\mathrm{x}, \mathrm{SDS} / \text { water }}$ and $\log K_{\mathrm{W}}$ data through eq 7 . Analysis of the experimental data in accordance with the Abraham model yielded the following correlation equation:

$$
\begin{gathered}
\log K_{\mathrm{SDS} / \mathrm{gas}}=-0.299(0.073)-0.087(0.086) \mathbf{E}+ \\
1.492(0.091) \mathbf{S}+3.374(0.081) \mathbf{A}+2.690(0.112) \mathbf{B}^{\circ}+ \\
\\
0.684(0.025) \mathbf{L}(12) \\
\left(N=142, R^{2}=0.992, \mathrm{SD}=0.223, F=3226.31\right)
\end{gathered}
$$

Preliminary regression analysis again identified heptane, octane, nonane, decane, and 1-decanol as outliers, and these five solutes were left out of the database used to generate eq 12. The statistics of eq 12 are quite good with a squared correlation coefficient of $R^{2}=0.992$ and a standard deviation of $\mathrm{SD}=0.223$ for a data set that covers a range of $13 \mathrm{log}$ units. In comparison, eq 6 , which uses the $\mathbf{V}$ solute descriptor, had a standard deviation of $\mathrm{SD}=0.31$. Part, though not all, of the reduced standard deviation for eq 12 is due to the use of the larger database combined with the removal of the four alkane solutes (heptane, octane, nonane, and decane) from the regression analyses. A slightly larger standard deviation of $\mathrm{SD}=0.243$ was obtained when the correlation was derived using only the $104 K_{\mathrm{SDS} / \text { gas }}$ values in the Bel'skii database. The fore-mentioned calculations clearly show that the Abraham model for solute transfer from 


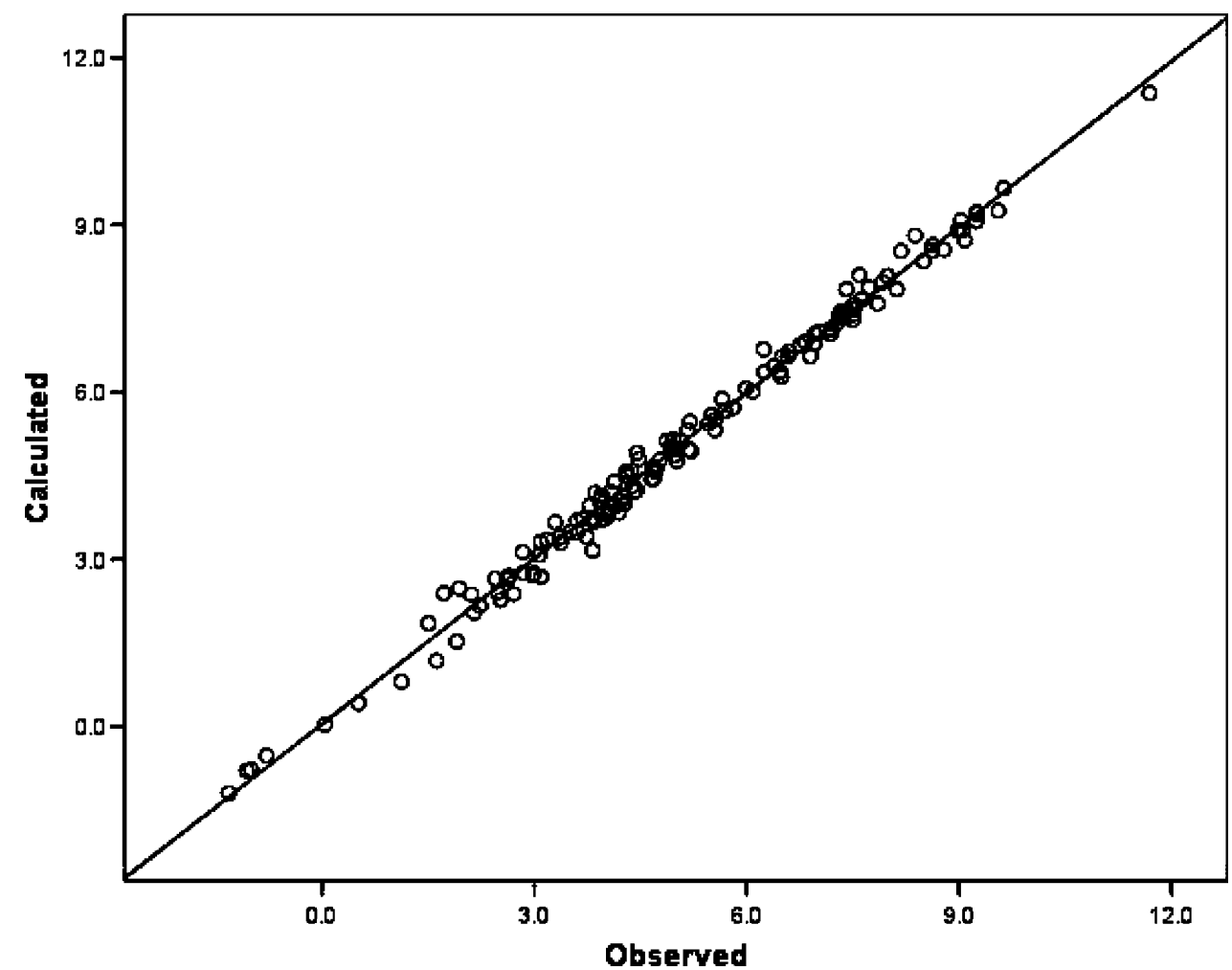

Figure 2. Experimental $\log K_{\mathrm{SDS} / \mathrm{gas}}$ data vs the calculated values based on eq 12 .

the gas phase is the more appropriate form of the two Abraham LFER equations for correlating $K_{\mathrm{SDS} / \text { water }}$ data. Figure 2 compares the calculated values of $\log K_{\mathrm{SDS} / \text { water }}$ based on eq 12 against the observed values. To our knowledge, there has been only one attempt to correlate $K_{\mathrm{SDS} / \text { water }}$ data, and that attempt also involved the Abraham model. ${ }^{5}$ Unlike Bel'skii, ${ }^{5}$ we used the form of the Abraham model that was developed for solute transfer from the gas phase to a condensed phase.

In order to assess the predictive ability of eq 12 we divided the 142 data points into a training set and a test set by allowing the SPSS software to randomly select half of experimental values. The selected data points became the training set, and the compounds that were left served as the test set. Analysis of the experimental data in the training set gave

$$
\begin{array}{r}
\log K_{\mathrm{SDS} / \mathrm{gas}}=-0.378(0.073)+0.046(0.125) \mathbf{E}+ \\
1.392(0.134) \mathbf{S}+3.391(0.127) \mathbf{A}+2.789(0.167) \mathbf{B}^{\circ}+ \\
0.685(0.034) \mathbf{L}
\end{array}
$$$$
\left(N=71, R^{2}=0.992, \mathrm{SD}=0.224, F=1718.99\right)
$$

There is very little difference in the equation coefficients for the full data set and training data set correlations. The training set correlation was then used to predict $\log K_{\mathrm{SDS} / \text { gas }}$ values for the 71 compounds in the test set. For the predicted and experimental values, we find that $\mathrm{SD}=0.2534$, AAE (average absolute error) $=0.1893$, and the $\mathrm{AE}$ (average error) $=-0.0009$. There is therefore very little bias in the predictions using eq 13 with $\mathrm{AE}$ equal to -0.0009 .

Micellar electrokinetic chromatography (MEKC) provides a convenient experimental method for determining waterto-micelle partition coefficients. The measured retention factor determined using a micellar surfactant pseudostation- ary phase is directly proportional to the partition coefficient. Several published studies have documented that the MEKC retention factor on a micellar SDS pseudostationary phase can be mathematically described by the Abraham model; however, none of the studies combined experimental $P_{\mathrm{x}, \mathrm{SDS} / \text { water }}$ and MEKC retention factors into a single correlation. Our search of the published literature found more than 250 experimental retention factors from eight studies, plus waterto-SDS partition coefficient data for 20 solutes that had been measured by a headspace chromatographic technique. Combined with the experimental $\log P_{\mathrm{x}, \mathrm{SDS} / \text { water }}$ data from Table 1 , we were able to build a database of 476 numerical values for regression analyses. To account for differences in experimental conditions, an indicator variable descriptor, I, was introduced for each of the eight chromatographic studies. A ninth indicator descriptor, $\mathbf{I}_{\text {Carr }}$, was used for the headspace chromatographic data ${ }^{33}$ because the numerical values of the partition coefficients were referenced to a different thermodynamic standard state. Each indicator descriptor was set equal to unity for all experimental values for the given study and set equal to zero otherwise. Analysis of the experimental data in Tables 1 and S1 yielded the following correlation

$$
\begin{gathered}
\log \left(P_{\mathrm{x}, \mathrm{SDS} / \text { water }} \text { or } k_{\mathrm{SDS}, \mathrm{MEKC}}\right)=1.310(0.031)+ \\
0.401(0.028) \mathbf{E}-0.441(0.032) \mathbf{S}-0.211(0.029) \mathbf{A}- \\
1.748(0.038) \mathbf{B}^{\circ}+2.936(0.035) \mathbf{V}- \\
1.122(0.038) \mathbf{I}_{\text {Carr }}-3.074(0.032) \mathbf{I}_{\text {MEKC-Roses }}- \\
3.174(0.028) \mathbf{I}_{\text {MEKC-Dorsey }}-3.182(0.054) \mathbf{I}_{\text {MEKC-Foley }}- \\
3.137(0.051) \mathbf{I}_{\text {MEKC-Muljeelaer }}- \\
3.208(0.023) \mathbf{I}_{\text {MEKC-Kelly }}-3.097(0.028) \mathbf{I}_{\text {MEKC-Poole }}- \\
3.111(0.031) \mathbf{I}_{\text {MEKC-Testa }}-3.247(0.030) \mathbf{I}_{\text {MEKC-Wall }}
\end{gathered}
$$




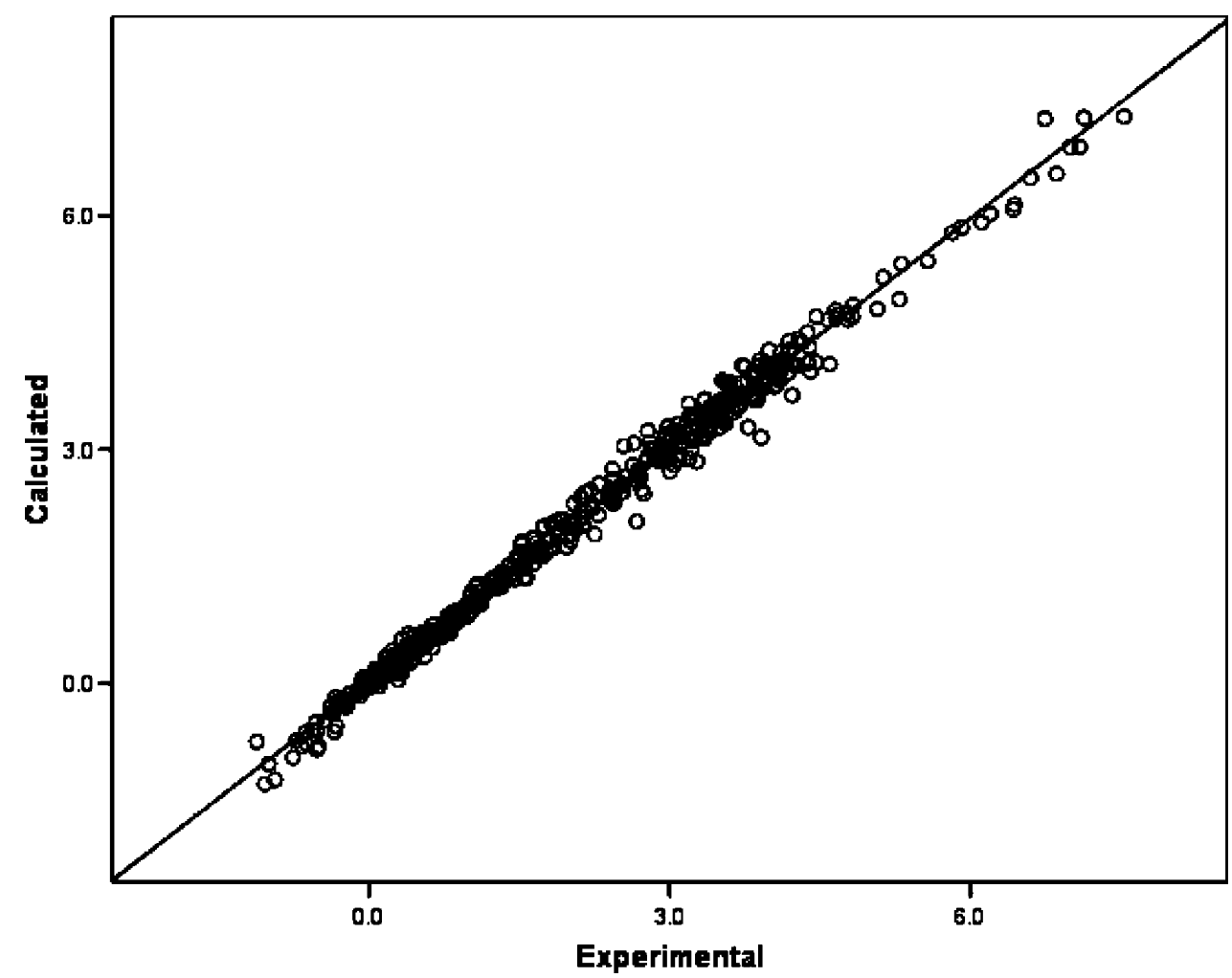

Figure 3. Experimental $\log \left(P_{\mathrm{x}, \mathrm{SDS} / \mathrm{water}}\right.$ or $\left.k_{\mathrm{SDS}, \mathrm{MEKC}}\right)$ data vs the calculated values based on eq 14 .

$$
\left(N=486, R^{2}=0.992, \mathrm{SD}=0.154, F=4106.65\right)
$$

The statistics of the eq 14 are quite good for a data set covering $8.65 \mathrm{log}$ units. A graphical comparison of the observed versus calculated values is shown in Figure 3. Careful examination of eqs 10 and 14 reveals that the SDS micellar solvent equation coefficients $(c, e, s, a, b$, and $v)$ are virtually identical for both correlations as would be expected if the measured retention factor truly represents solute partitioning from water to the SDS micellar phase. The molecular interactions between the dissolved solute and the SDS micellar phase is the same for both $P_{\mathrm{x}, \mathrm{SDS} / \text { water }}$ and $k_{\mathrm{MEKC}}$ data.

The differences in the experimental conditions employed by the different research groups are reflected by the variation in the eight indicator variable coefficients. The numerical values of the eight MEKC coefficients span a fairly narrow range, from -3.073 for $\mathbf{I}_{\text {MEKC-Roses }}$ coefficient to -3.245 for $\mathbf{I}_{\text {MEKC-Wall }}$ coefficient. For correlation purposes the eight MEKC coefficients are identical. We analyzed the 486 data points one final time

$$
\begin{gathered}
\log \left(P_{\mathrm{x}, \mathrm{SDS} / \text { water }} \text { or } k_{\mathrm{SDS}, \mathrm{MEKC}}\right)=1.334(0.032)+ \\
0.391(0.028) \mathbf{E}-0.419(0.033) \mathbf{S}-0.229(0.026) \mathbf{A}- \\
1.751(0.039) \mathbf{B}^{\mathbf{o}}+2.909(0.035) \mathbf{V}- \\
1.127(0.039) \mathbf{I}_{\mathrm{Carr}}-3.163(0.016) \mathbf{I}_{\text {MEKC }} \\
\left(N=486, R^{2}=0.991, \mathrm{SD}=0.160, F=7765.53\right)
\end{gathered}
$$

with a single $\mathbf{I}_{\text {MEKC }}$ indicator variable to represent all of the MEKC retention data. Eq 15 provides a very good correlation of all of the water-to-SDS micellar partition coefficients and MEKC retention data.
Gil-Agustí et al. ${ }^{41}$ have compared a number of SDSalcohol systems using an Abraham LFER and indicator variables for the various systems; the stratagem we have followed is exactly the same. Gil-Agustí also compared the Abraham system coefficients for SDS systems with those for various HPLC systems and water-solvent partition coefficients. The system coefficients in all these cases contain information on the partition of solutes between two phases, for example between water and an SDS micelle or between the mobile phase and the stationary phase in an HPLC system. The system coefficients thus encode information on the differential interaction of the solute with the two phases, that is the difference in solute-solvent interactions with each of the phases. However, when an Abraham equation is applied to partition from the gas phase to a given solvent system, the coefficients encode information on the solutesolvent system only. These coefficients for transfer from the gas phase are therefore much easier to interpret, and so we have taken advantage of the large data set we have assembled on gas to SDS transfer, and the consequent eq 12 to compare coefficients with other gas to solvent phase transfers.

The phases and their coefficients are in Table 3 and include a number of room-temperature ionic liquids, RTILs, that we have previously characterized ${ }^{42}$ as well as a number of representative organic solvents. ${ }^{42}$ The $a$ - and $b$-coefficients for the SDS phase are quite large and indicate that the interactions of the phase with basic and acidic solutes will be quite large. A useful way of comparing sets of coefficients is to carry out a principal components analysis, PCA, on the coefficients $e$ to $l$. The five columns of coefficients are transformed into five PCs. In the present case, the first two PCs contain $76 \%$ of the total information, so that a plot of the scores of the first two PCs will give an indication of how close are the sets of coefficients. As can be seen from 
Table 3. System Coefficients for Gas to Condensed Phase Processes $^{a}$

\begin{tabular}{|c|c|c|c|c|c|c|c|}
\hline phase & no. & $c$ & $e$ & $s$ & $a$ & $b$ & $l$ \\
\hline S & 1 & 99 & -0 . & 2 & 3.374 & 2.690 & 068 \\
\hline $\mathrm{Im}+$ & 2 & & & & & & \\
\hline+ & 3 & .170 & -0.1 & & & 0.429 & $\pi$ \\
\hline & 4 & 0.288 & 0.115 & & & 0.7 & 62 \\
\hline & 5 & -0.268 & -0.100 & & & & 0.72 \\
\hline & 6 & -0.611 & 0.487 & 2.484 & & & 0.60 \\
\hline & 7 & -1 & -0.087 & & & & 63 \\
\hline & 8 & -0.439 & & & & & .62 \\
\hline & 9 & - & & & & & 65 \\
\hline & 10 & & - & & & & 97 \\
\hline & & & & & & & 00 \\
\hline & 1 & 0.2 & & & & & 04 \\
\hline & 13 & & & & & & 50 \\
\hline & 1 & & & & & & 0.936 \\
\hline & & & & & & & .99 \\
\hline & & & -0 & & & & .9 \\
\hline & & & & & & & .86 \\
\hline & 18 & 0 & & & & 0. & 0.91 \\
\hline & 19 & -0.0 & & & & & 0.73 \\
\hline & 20 & & & & & & .83 \\
\hline & 21 & - & & & & & 81 \\
\hline & 22 & -( & & & & & 0.73 \\
\hline & 23 & 1 & -0 & & & & 1.01 \\
\hline & 24 & & -0 . & & & & 1.0 \\
\hline & 25 & -0.295 & & & & 0. & 0.91 \\
\hline doro & 26 & -0.133 & & & & 4.461 & 0.63 \\
\hline & 27 & & & & & & \\
\hline & 28 & & -0 . & & & & 0.9 \\
\hline & 2 & & & & & & \\
\hline & 30 & -1.271 & 0.822 & 2.743 & 3.904 & 4.814 & -0.2 \\
\hline
\end{tabular}

${ }^{a}$ Abbreviations: 1-methyl-3-butylimidazolium bis(trifluoromethylsulfonyl)imide $\left([\mathrm{MBIm}]^{+}\left[\mathrm{Tf}_{2} \mathrm{~N}\right]^{-}\right)$; 1-methyl-3-hexylimidazolium bis(trifluoromethylsulfonyl)imide $\left([\mathrm{MHIm}]^{+}\left[\mathrm{Tf}_{2} \mathrm{~N}\right]^{-}\right)$; trimethylbutylammonium bis(trifluoromethylsulfonyl)imide $\left(\left[\mathrm{M}_{3} \mathrm{BAm}\right]^{+}\left[\mathrm{Tf}_{2} \mathrm{~N}\right]^{-}\right)$; 1-methyl3-octylimidazolum tetrafluoroborate $\left([\mathrm{MOIm}]^{+}\left[\mathrm{BF}_{4}\right]^{-}\right)$; 4-methyl- $N$ butylpyridinium tetrafluoroborate ([MBPy $\left.]^{+}\left[\mathrm{BF}_{4}\right]^{-}\right)$; 1-methyl-3butylimidazolium hexafluorophosphate $\left([\mathrm{MBIm}]^{+}\left[\mathrm{PF}_{6}\right]^{-}\right)$; 1-methyl3-ethylimidazolium bis(trifluoromethylsulfonyl)imide $\left([\mathrm{MEIM}]^{+}\left[\mathrm{Tf}_{2} \mathrm{~N}\right]^{-}\right.$); 1,2-dimethyl-3-ethylimidazolium bis(trifluoromethylsulfonyl)imide $\left(\left[\mathrm{M}_{2} \mathrm{EIM}\right]^{+}\left[\mathrm{Tf}_{2} \mathrm{~N}\right]^{-}\right)$.

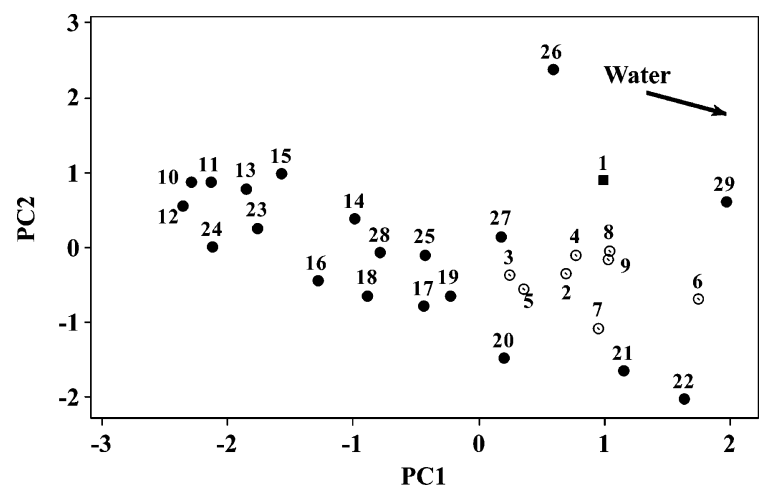

Figure 4. A plot of the scores of $\mathrm{PC} 2$ against $\mathrm{PC} 1$ for the coefficients of the phases listed in Table 3. Points numbered as in Table 3. The point for water has been left out, as it is well off scale. SDS $\mathbf{\square}$; RTILs $\bigcirc$; remaining phases $\bullet$

Figure 4, the SDS system, no. 1, is quite far from organic solvents that are nonpolar or of only medium polarity. The nearest are methanol, no. 27, and ethylene glycol, no. 29, indicating how polar is the SDS system. Perhaps surprisingly, the SDS system is quite close to the RTILs, nos. 2-9, especially nos. $2,4,8$, and 9 . It is difficult to think of any other methodology that could yield such an intriguing result.
Table 4. Selective Solubility of Gaseous Solutes in Various Phases as $\alpha=\log K$ (solute 1$)-\log K$ (solute 2)

\begin{tabular}{|c|c|c|c|}
\hline $\begin{array}{lr}\text { solute } 1 & \text { benzene } \\
\text { solute } 2 & \text { cyclohexane }\end{array}$ & $\begin{array}{r}\text { butylamine } \\
\text { bromobutane }\end{array}$ & \multicolumn{2}{|c|}{$\begin{array}{r}\text { butan-1-ol } \\
\text { methyl butyl ether }\end{array}$} \\
\hline system & \multicolumn{3}{|c|}{ selectivity, $\alpha$} \\
\hline SDS & 0.855 & 1.794 & 1.555 \\
\hline$\left([\mathrm{MBIm}]^{+}\left[\mathrm{Tf}_{2} \mathrm{~N}\right]^{-}\right)$ & 0.891 & 0.607 & 1.279 \\
\hline$\left([\mathrm{MHIm}]^{+}\left[\mathrm{Tf}_{2} \mathrm{~N}\right]^{-}\right)$ & 0.773 & 0.464 & 1.102 \\
\hline$\left(\left[\mathrm{M}_{3} \mathrm{BAm}\right]^{+}\left[\mathrm{Tf}_{2} \mathrm{~N}\right]^{-}\right)$ & 0.884 & 0.579 & 1.158 \\
\hline$\left([\mathrm{MOIm}]^{+}\left[\mathrm{BF}_{4}\right]^{-}\right)$ & 0.660 & 0.661 & 1.458 \\
\hline$\left([\mathrm{MBPy}]^{+}\left[\mathrm{BF}_{4}\right]^{-}\right)$ & 1.162 & 0.589 & 1.678 \\
\hline$\left([\mathrm{MBIm}]^{+}\left[\mathrm{PF}_{6}\right]^{-}\right)$ & 1.074 & 0.383 & 1.468 \\
\hline$\left([\mathrm{MEIM}]^{+}\left[\mathrm{Tf}_{2} \mathrm{~N}\right]^{-}\right)$ & 1.035 & 0.721 & 1.223 \\
\hline$\left(\left[\mathrm{M}_{2} \mathrm{EIM}\right]^{+}\left[\mathrm{Tf}_{2} \mathrm{~N}\right]^{-}\right)$ & 1.060 & 0.622 & 1.204 \\
\hline hexane & -0.226 & 0.022 & -0.086 \\
\hline hexadecane & -0.178 & -0.002 & -0.057 \\
\hline tetrachloromethane & -0.086 & 0.018 & -0.036 \\
\hline carbon disulfide & -0.026 & 0.004 & 0.028 \\
\hline 1,2-dichloroethane & 0.494 & 0.412 & 0.433 \\
\hline chloroform & 0.386 & 0.728 & 0.173 \\
\hline ether & 0.046 & 0.486 & 1.119 \\
\hline acetone & 0.412 & 0.521 & 1.369 \\
\hline ethyl acetate & 0.260 & 0.455 & 1.192 \\
\hline acetonitrile & 0.779 & 0.498 & 1.058 \\
\hline dimethylformamide & 0.721 & 0.589 & 1.807 \\
\hline$N$-methylpyrrolidinone & 0.860 & 0.667 & 2.259 \\
\hline dimethylsulfoxide & 1.090 & 0.715 & 2.485 \\
\hline toluene & 0.160 & 0.106 & 0.239 \\
\hline chlorobenzene & 0.173 & 0.072 & 0.190 \\
\hline nitrobenzene & 0.632 & 0.278 & 0.732 \\
\hline 2,2,2-trifluoroethanol & 0.937 & 2.502 & 0.983 \\
\hline methanol & 0.491 & 1.264 & 1.544 \\
\hline octan-1-ol & 0.104 & 0.915 & 1.356 \\
\hline ethylene glycol & 0.940 & 1.859 & 2.058 \\
\hline water & 2.115 & 2.730 & 2.263 \\
\hline
\end{tabular}

The closeness of the SDS system to the RTILs indicates that solvation of gaseous solutes in these various systems is quite similar but does not give precise information as to the selectivity of the systems for various types of solute. A considerable advantage of the present methodology is that it is very easy to calculate gas to solvent partition, as $\log K$ values for a large number of solutes. Then for two solutes in a given phase, the selectivity of the phase can be obtained quantitatively as

$$
\alpha=\log K(\text { solute } 1)-\log K(\text { solute } 2)
$$

In Table 4 are given values of $\alpha$ for a number of pairs of solute of about the same size. For benzene and cyclohexane the SDS system is reasonably selective toward benzeneabout the same as some of the RTILs but somewhat less than the polar organic phases. For the butylamine and bromobutane solute pair, the SDS system is very selective toward the organic base, much more than the RTILs, and exceeded in selectivity only by the organic solvents that are very polar. The final pair of solutes includes the hydrogen bond acid, butan-1-ol, and the corresponding methyl ether; again, the SDS system shows good selectivity.

We can therefore predict that the SDS system will show as good a selectivity toward aromatic compounds, hydrogen bond bases, and hydrogen bond acids as do the RTILs. Since the SDS system is largely aqueous, it represents a very environmentally useful selective solvent system. The organic solvents that are more selective than the SDS system are all environmentally hazardous, while water, the most selective phase, suffers from poor solubility for the less polar solutes. 
Supporting Information Available: Micellar electrokinetic chromatographic retention factor data for organic solutes determined using a micellar SDS surfactant pseudostationary phase and numerical values of the solute descriptors for the compounds studied (Table S1). This material is available free of charge via the Internet at http://pubs.acs.org

\section{REFERENCES AND NOTES}

(1) Sepulveda, L.; Lissi, E.; Quina, F. Interactions of neutral molecules with ionic micelles. Adv. Colloid Interface Sci. 1986, 25, 1-57.

(2) Abraham, M. H.; Chadha, H. S.; Dixon, J. P.; Rafols, C.; Treiner, C. Hydrogen bonding. Part 40. Factors that influence the distribution of solutes between water and sodium dodecyl sulfate micelles. J. Chem. Soc., Perkin Trans. 2 1995, 887-894.

(3) Abraham, M. H.; Chadha, H. S.; Dixon, J. P.; Rafols, C.; Treiner, C. Hydrogen bonding. Part 41. Factors that influence the distribution of solutes between water and hexadecylpyridinium chloride micelles. $J$. Chem. Soc., Perkin Trans. 2 1997, 19-24.

(4) Valsaraj, K. T.; Thibodeaux, L. J. On the estimation of micelle-water partition constants for solutes from their octanol-water partition constants, normal boiling points, aqueous solubilities, and group and bond contribution schemes. Sep. Sci. Technol. 1990, 25, 369-395.

(5) Bel'skii, V. E. Distribution of nonionic compounds between the gas phase and sodium dodecyl sulfate micelles. Russ. Chem. Bull. 2000, $49,251-255$.

(6) Abraham, M. H.; Acree, W. E., Jr. Comparative analysis of solvation and selectivity in room temperature ionic liquids using the Abraham linear free energy relationship. Green Chem. 2006, 8, 906-915.

(7) Roses, M.; Rafols, C.; Bosch, E.; Martinez, A. M.; Abraham, M. H Solute-solvent interactions in micellar electrokinetic chromatography. Characterization of sodium dodecyl sulfate-Brij 35 micellar systems for quantitative structure-activity relationship modeling. J. Chromatogr., A 1999, 845, 217-226.

(8) Herbert, B. J.; Dorsey, J. G. n-Octanol-water partition coefficient estimation by micellar electrokinetic capillary chromatography. Anal. Chem. 1995, 67, 744-749.

(9) Ahuja, E. S.; Foley, J. P. Influence of dodecyl sulfate counterion on efficiency, selectivity, retention, elution range, and resolution in micellar electrokinetic chromatography. Anal Chem. 1995, 67, 23152324.

(10) Muijselaar, P. G. H. M.; Claessens, H. A.; Cramers, C. A. Application of the retention index concept in micellar electrokinetic capillary chromatography. Anal. Chem. 1994, 66, 635-644.

(11) Kelly, K. A.; Burns, S. T.; Khaledi, M. G. Prediction of retention in micellar electrokinetic chromatography from solute structure. 1. Sodium dodecyl sulfate micelles. Anal. Chem. 2001, 73, 6057-6062.

(12) Poole, S. K.; Poole, C. F. Characterization of surfactant selectivity in micellar electrokinetic chromatography. Analyst 1997, 122, 267-274.

(13) Taillardat-Bertschinger, A.; Carrupt, P.-A.; Testa, B. The relative partitioning of neutral and ionized compounds in sodium dodecyl sulfate micelles measured by micellar electrokinetic capillary chromatography. Eur. J. Pharm. Sci. 2002, 15, 225-234.

(14) Wall, W. E.; Allen, D. J.; Denson, K. D.; Love, G. I.; Smith, J. T. Explorations of alkyl polyols as "class I" organic modifiers to adjust selectivity in micellar electrokinetic capillary chromatography. Electrophoresis 1999, 20, 2390-2399.

(15) Bel'skii, V. E. The influence of the structure of organic compounds on their solubilization by sodium dodecyl sulfate micelles. Russ. Chem. Bull. 1999, 48, 864-869.

(16) Fuangswasdi, A.; Charoensaeng, A.; Sabatini, D. A.; Scamehorn, J. F.; Acosta, E. J.; Osathaphan, K.; Khaodhiar, S. Mixtures of anionic and cationic surfactants with single and twin head groups: solubilization and adsolubilization of styrene and ethylcyclohexane. $J$. Surfactants Deterg. 2006, 9, 29-37.

(17) Shimotori, T.; Arnold, W. A. Measurement and estimation of Henry's law constants of chlorinated ethylenes in aqueous surfactant solutions. J. Chem. Eng. Data 2003, 48, 253-261.

(18) Vane, L. M.; Giroux, E. L. Henry's law constants and micellar partitioning of volatile organic compounds in surfactant solutions. $J$. Chem. Eng. Data 2000, 45, 38-47.

(19) Manabe, M.; Tokunaga, A.; Kawamura, H.; Katsuura, H.; Shiomi, M.; Hiramatsu, K. The counterion releasing effect and the partition coefficient of branched alkanols in ionic micellar solution. Colloid Polym. Sci. 2002, 280, 929-935.
(20) Fortuny, M.; Graillat, C.; McKenna, T. F. A new technique for the experimental measurement of monomer partition coefficients. Macromol. Chem. Phys. 2004, 205, 1309-1319.

(21) Hoiland, H.; Gjerde, M. I.; Mo, C.; Lie, E. Solubilization of alcohols in SDS and TTAB from isentropic partial molar compressibilities and solubilities. Colloids Surf., A 2001, 183-185, 651-660.

(22) Naeem, K.; Waheed, A.; Shah, S. S. Conductometric study of short chain alkanones and alkyl acetates within anionic micelles. J. Surf. Sci. Technol. 1997, 13, 153-158.

(23) McMahon, C. A.; Hawrylak, B.; Marangoni, D. G.; Palepu, R. Calorimetric and NMR investigations of the micellar properties of sodium dodecyl sulfate in aqueous mixtures of isomeric butanediols. Langmuir 1999, 15, 429-436.

(24) Hussam, A.; Basu, S. C.; Hixon, M.; Olumee, Z. General method for the study of solute-surfactant association equilibria of volatile solutes by headspace gas chromatography. Anal. Chem. 1995, 67, 1459-1464.

(25) Paria, S.; Yuet, P. K. Solubilization of naphthalene by pure and mixed surfactants. Ind. Eng. Chem. Res. 2006, 45, 3552-3558.

(26) Liu, G. G.; Roy, D.; Rosen, M. J. A simple method to estimate the surfactant micelle-water distribution coefficients of aromatic hydrocarbons. Langmuir 2000, 16, 3595-3605.

(27) Tokuoka, Y.; Uchiyama, H.; Abe, M.; Christian, S. D. Solubilization of some synthetic perfumes by anionic-nonionic mixed surfactant systems. Part 1. Langmuir 1995, 11, 725-729.

(28) Dulfer, W. J.; Bakker, M. W. C.; Govers, H. A. J. Micellar solubility and micelle/water partitioning of polychlorinated biphenyls in solutions of sodium dodecyl sulfate. Environ. Sci. Technol. 1995, 29, 985992.

(29) Khan, M. N. Spectrophotometric determination of anionic micellar binding constants of ionized and non-ionized phenyl and methyl salicylates. J. Phys. Org. Chem. 1996, 9, 295-300.

(30) Shah, S. W. H.; Naseem, B.; Naeem, K.; Malik, Q. M.; Shah, S. S. Incorporation of aromatic acids into sodium dodecyl sulfate micelles: a UV-spectroscopic analysis. J. Surf. Sci. Technol. 2001, 17, 49-55.

(31) Bertolotti, S. G.; Garcia, N. A.; Gsponer, H. E. Micellar binding of polychlorophenols. J. Colloid Interface Sci. 1989, 129, 406-413.

(32) Katsuta, S.; Saitoh, K. A micellar electrokinetic chromatographic method for determination of solubilization isotherms in surfactant micelles. Anal. Chem. 1998, 70, 1389-1393.

(33) Vitha, M. F.; Weckwerth, J. D.; Odland, K.; Dema, V.; Carr, P. W. Study of the polarity and hydrogen bond ability of sodium dodecyl sulfate micelles by the Kamlet-Taft solvatochromic comparison method. J. Phys. Chem. 1996, 100, 18823-18828.

(34) Abraham, M. H.; Ibrahim, A.; Zissimos, A. M. Determination of sets of solute descriptors from chromatographic measurements. J. Chromatogr., A 2004, 1037, 29-47.

(35) Pharma Algorithms, ADME Boxes, Version 3.0; Pharma Algorithms Inc.: Toronto, Ontario, Canada, 2006.

(36) Platts, J. A.; Butina, D.; Abraham, M. H.; Hersey, A. Estimation of molecular linear free energy relation descriptors using a group contribution approach. J. Chem. Inf. Comput. Sci. 1999, 39, 835845.

(37) Jover, J.; Bosque, R.; Sales, J. Determination of Abraham solute parameters from molecular structure. J. Chem. Inf. Comput. Sci. 2004, 44, 1098-1106.

(38) Arey, J. S.; Green, W. H., Jr.; Gschwend, P. M. The electrostatic origin of Abraham's solute polarity parameter. J. Phys. Chem. B 2005, 109 , 7564-7573.

(39) Mutelet, F.; Rogalski, M. Experimental determination and prediction of the gas-liquid n-hexadecane partition coefficients. J. Chromatogr., A 2001, 923, 153-163.

(40) Treiner, C.; Mannebach, M. H. Correlation analysis of solubilization data in aqueous cationic and anionic micellar solutions: case of the halocarbons. J. Colloid Interface Sci. 1987, 118, 243-251.

(41) Gil-Agustí, M.; Esteve-Romero, J.; Abraham, M. H. Solute-solvent interactionsin micellar liquid chromatography. Characterization of hybrid micellar systems of sodium dodecyl sulfate-pentanol. $J$. Chromatogr. A 2006, 1117, 47-55.

(42) Abraham, M. H.; Acree, W. E., Jr. Comparative analysis of solvation andselectivity in room temperature ionic liquids using the Abraham linear free energy relationship. Green Chem. 2006, 6, 906-915.

CI700174Q 